\title{
Multi-colour optical monitoring of eight red blazars ${ }^{\star}$
}

\author{
M. F. Gu ${ }^{1,2,3}$, C.-U. Lee ${ }^{1}$, S. Pak ${ }^{4}$, H. S. Yim ${ }^{1}$, and A. B. Fletcher ${ }^{1,2}$ \\ 1 Korea Astronomy and Space Science Institute, 61-1 Whaam-dong, Yuseong-gu, Daejeon 305-348, Republic of Korea \\ e-mail: gumf@shao.ac.cn \\ 2 Shanghai Astronomical Observatory, Chinese Academy of Sciences, 80 Nandan Road, Shanghai 200030, PR China \\ 3 National Astronomical Observatories, Chinese Academy of Sciences, Beijing 100012, PR China \\ 4 Dept. of Astronomy and Space Science, Kyung Hee University, Kyunggi-do 446-701, Korea
}

Received 29 September 2005 / Accepted 15 December 2005

\begin{abstract}
Context. We present the observational results of multi-colour optical monitoring of eight red blazars from 2003 September to 2004 February. Aims. The aim of our monitoring is to investigate the spectral variability as well as the flux variations at short and long time scales. Methods. The observations were carried out using the $1.0 \mathrm{~m}$ robotic telescope of Mt. Lemmon Optical Astronomy Observatory, in Arizona, USA, the $0.6 \mathrm{~m}$ telescope of Sobaeksan Optical Astronomy Observatory and the $1.8 \mathrm{~m}$ telescope of Bohyunsan Optical Astronomy Observatory, in the Republic of Korea.

Results. During the observations, all sources show strong flux variations with amplitudes of larger than 0.5 mag. Variations with amplitudes of over 1 mag are found in four sources. Intraday variations with amplitudes larger than 0.15 mag, and a rapid brightness increase with a rate of $\sim 0.2$ mag per day in four days, are detected in S5 0716+71. We investigate the relationship between the colour index and source brightness for each source. We find that two out of three FSRQs tend to be redder when they are brighter, and, conversely, all BL Lac objects tend to be bluer. In particular, we find a significant anti-correlation between the $V-I$ colour index and $R$ magnitude for 3C 454.3. This implies that the spectrum became steeper when the source was brighter, which is opposite to the common trend for blazars. In contrast, significant positive correlations are found in 3C 66A, S5 0716+71, and BL Lac. However, there are only very weak correlations for PKS $0735+17$ and OJ 287. Conclusions. We propose that the different relative contributions of the thermal versus non-thermal radiation to the optical emission may be responsible for the different trends of the colour index with brightness in FSRQs and BL Lac objects.
\end{abstract}

Key words. galaxies: active - galaxies: BL Lacertae objects: general - galaxies: quasars: general - galaxies: photometry

\section{Introduction}

Blazars, including BL Lac objects and flat-spectrum radio quasars (FSRQs), are the most extreme class of active galactic nuclei (AGNs), characterized by strong and rapid variability, high polarization, and apparent superluminal motion. These extreme properties are generally interpreted as a consequence of non-thermal emission from a relativistic jet oriented close to the line of sight. As such, they represent a fortuitous natural laboratory with which to study the physical properties of jets, and, ultimately, the mechanisms of energy extraction from the central supermassive black holes.

In the field of AGN observations, one of the most important discoveries in the last decade has been that blazars emit a substantial fraction, sometimes most of their power, at $\gamma$-ray energies ( $\mathrm{GeV}$ and TeV, e.g. Ulrich et al. 1997; Catanese \& Weeks 1999). The $\gamma$-ray emission of blazars indicates a double-peak

* Table 2 is only available in electronic form at the CDS via anonymous ftp to cdsarc.u-strasbg.fr (130.79.125.5) or via http: //cdsweb.u-strasbg.fr/cgi-bin/qcat? J/A+A/450/39 structure in the overall spectral energy distribution (SED), with two broad spectral components. The first, lower frequency component is generally interpreted as being due to synchrotron emission, and the second, higher frequency one as being due to inverse Compton emission. Although different blazars have different peak frequencies, the two peaks in their SEDs are separated by approximately the same amount, i.e. 8-10 decades in frequency (Fossati et al. 1998). According to the different peak frequencies, blazars are divided into two subclasses: the low-energy-peaked blazars (red blazars), which have synchrotron peaks in the IR/optical range, and the high-energypeaked blazars (blue blazars), which have synchrotron peaks at $\mathrm{UV} / \mathrm{X}$-ray energies. All of the $\mathrm{TeV} \gamma$-ray-loud AGNs are blue blazars, while all strong GeV $\gamma$-ray AGNs are red blazars (Mattox et al. 1997; Hartman et al. 1999; Bai \& Lee 2001).

Although a point of agreement about the second component is that the $\gamma$-rays of blazars are produced in relativistic jets by inverse Compton scattering, the origin of the seed photons (optical/IR), the location and size of the emitting region, and the degree of relativistic beaming of the high-energy radiation, 
Table 1. List of the eight blazar sources in the optical monitoring sample.

\begin{tabular}{|c|c|c|c|c|c|c|}
\hline Object & Name & Class. & Filters & Observatory & $\gamma$-detected & Comparison Stars \\
\hline $0219+428$ & $3 \mathrm{C} 66 \mathrm{~A}$ & BL Lac & $B V R I$ & $\mathrm{~L}, \mathrm{~S}$ & Yes & Fiorucci \& Tosti (1996) $)^{b}$ \\
\hline 0420-014 & PKS 0420-01 & FSRQ & $V R I$ & $\mathrm{~L}, \mathrm{~S}$ & Yes & Smith \& Balonek (1998) \\
\hline $0716+714$ & S5 $0716+71$ & BL Lac & $B V R I$ & $\mathrm{~B}, \mathrm{~L}, \mathrm{~S}$ & Yes & Villata et al. $(1998 \mathrm{a})^{a}$ \\
\hline $0735+178$ & PKS 0735+17 & BL Lac & $V R I$ & $\mathrm{~L}, \mathrm{~S}$ & Yes & Smith et al. (1985) \\
\hline $0851+202$ & OJ 287 & BL Lac & $V R I$ & $\mathrm{~L}, \mathrm{~S}$ & Yes & Fiorucci \& Tosti (1996) \\
\hline $1641+399$ & $3 \mathrm{C} 345$ & FSRQ & $V R I$ & $\mathrm{~L}, \mathrm{~S}$ & No & Smith et al. (1985) \\
\hline $2200+420$ & BL Lac & BL Lac & $V R I$ & $\mathrm{~L}, \mathrm{~S}$ & Yes & Fiorucci \& Tosti $(1996)^{b}$ \\
\hline $2251+158$ & $3 C 454.3$ & FSRQ & $V R I$ & $\mathrm{~L}, \mathrm{~S}$ & Yes & Fiorucci et al. (1998) \\
\hline
\end{tabular}

${ }^{a}$ I-data from Ghisellini et al. (1997).

${ }^{b}$ Magnitudes from González-Pérez et al. (2001).

are all still unknown. Seed photons may come from the jet itself (Synchrotron Self-Compton model), from an accretion disk around a supermassive black hole at the base of the jet, or else from photons of the broad emission line region (e.g. Wehrle et al. 1998; Collmar et al. 2000). Some models suggest that the flares of $\gamma$-rays in blazars are caused by the increase and decrease of the bulk Lorentz factor, while other models suggest changes in both the injection rate and the spectral shape of the injected electrons (Mukherjee et al. 1997; Hartman et al. 2001a; Pian et al. 2002; Villata et al. 2002).

Simultaneous multiwavelength observations have shown that both $\mathrm{TeV}$ and $\mathrm{GeV} \gamma$-ray blazars vary much more violently at and above their peaks, than below these peaks, and that flux variations at these two peaks are correlated, indicating that both components are produced by the same electron population in the relativistic jet (e.g. Ulrich et al. 1997; Sambruna et al. 2000; Hartman et al. 2001b). Since red blazars have synchrotron emission peaking in the IR/optical bands, observations at these wavelengths are thus crucial in understanding the nature of $\gamma$-ray emission in red blazars. The near-IR/optical bands are ideal for monitoring the flux and spectral variability, as well as to determine the frequencies and frequency shifts of the synchrotron peaks for red blazars. The flux and spectral variability, together with the synchrotron peak frequency and its shift, allow one to derive information on the radiating relativistic electrons, and on the emission region (Ghisellini et al. 1997). Moreover, the differences in the variability behaviour, in the IR/optical bands, of non- $\gamma$-ray-detected red blazars, as compared to those of the $\gamma$-ray-loud ones, may strongly constrain models for $\gamma$-ray emission.

The study of the properties of the SED is an excellent diagnostic tool for theoretical models. However, the availability of simultaneous multiwavelength observations is generally inadequate in tracing the time evolution for both the synchrotron and inverse Compton components. A relatively simple method is to investigate time evolution in a selected region of the spectra, such as in the optical. Although the optical region is narrow with respect to other spectral regions, it may provide a relatively large amount of information. In this case, the possible presence of other components in addition to the synchrotron emission, e.g. thermal emission from the accretion disk around the central engine, may complicate the situation. Nevertheless, statistical analysis of the optical spectral variability could enable us to constrain theoretical models; as claimed by Vagnetti et al. (2003), the spectral variability, even when restricted to the optical band, can be used to set limits on the relative contribution of the synchrotron component and the thermal component to the overall SED.

In this paper, we investigate the spectral variability, as well as the multi-band flux variability for eight typical red blazars, which consist of three FSRQs and five BL Lac objects. The spectral variability of the FSRQs is compared with that of the BL Lac objects. Based on analysis of the spectral variability, we attempt to derive information to constrain the theoretical models. The observations and data reduction are given in Sect. 2. The flux variability is shown in Sect. 3, and the spectral variability is described in Sect. 4. The discussions are given in Sect. 5, and the main results are summarized in Sect. 6.

\section{Observations and data reduction}

Multi-site photometric observations of eight blazars were carried out for a combined total of 50 nights, from 2003 September to 2004 February, using the $1.0 \mathrm{~m}$ robotic telescope of Mt. Lemmon Optical Astronomy Observatory (denoted as L in Table 1; Han et al. 2005), in Arizona, USA, for 33 nights, the $0.6 \mathrm{~m}$ telescope of Sobaeksan Optical Astronomy Observatory (denoted as $\mathrm{S}$ in Table 1) and the $1.8 \mathrm{~m}$ telescope of Bohyunsan Optical Astronomy Observatory (denoted as B in Table 1), in the Republic of Korea, for 14 and 3 nights, respectively. The observations were made in 5 sessions to investigate both long-term and intraday variations: 5 nights in September, 10 nights in October, 13 nights in November, 16 nights in December, 2003, and 6 nights in February, 2004. All telescopes were equipped with CCD cameras, and $U B V$ (Johnson) and $R I$ (Cousins) filter sets. LN2 cryogenic systems were used for both the $1.8 \mathrm{~m}$ and $0.6 \mathrm{~m}$ telescopes, while a thermoelectric cooling system was used for the $1.0 \mathrm{~m}$ telescope. These three telescopes cover different field of views: $22.2 \times 22.2 \mathrm{arcmin}$, $20.5 \times 20.5$ arcmin, and $11.7 \times 11.7$ arcmin for the $1.0 \mathrm{~m}, 0.6 \mathrm{~m}$, and $1.8 \mathrm{~m}$ telescopes, respectively. Considering the aperture sizes of the telescopes and the seeing conditions, different exposure times were applied. While observing, we tried to position each target object at the same location on the CCD surface, 
to within a few pixels, in order to achieve more efficient and accurate photometry. Sky flat images were taken at both dusk and dawn when available. Bias and dark images were taken after obtaining sky flat images.

All images were preprocessed with the standard procedures, using the $\mathrm{IRAF}^{1}$ software, including bias and dark subtraction, and flat-fielding. For images obtained from the $0.6 \mathrm{~m}$ telescope, the fringe pattern was carefully corrected. We used the APPHOT package in IRAF to measure the instrumental magnitudes of the blazars and comparison stars. The seeing variations during one night may cause variations of the instrumental magnitude, and there is a trend of the instrumental magnitude variations with the FWHM variations of a star from night to night (Clements \& Carini 2001). In order to correct the seeing variation effect during one night, and to try to include the "total" flux from objects when seeing varies (especially when seeing is poor), we set the radius in the aperture photometry to be proportional to the FWHM of bright, well-exposed stars in the image frames containing the objects, during one night (Lee et al. 2003). After experimenting with various aperture sizes, we set the aperture radius at $4 \times \mathrm{FWHM}$, the inner radius of the sky annulus at $5 \times$ FWHM, and the width of the sky annulus at 10 pixels, to maximize the $\mathrm{S} / \mathrm{N}$ ratio.

In Table 1, the source IAU name and the alias name are given in Cols. 1 and 2, respectively. The classification, filter sets, telescopes, and the detection in the $\gamma$-region for each source are shown in Cols. 3-6. The source magnitude is calibrated with respect to the standard stars, for which references are given in Col. 7 of Table 1. The observational errors are estimated from the rms differential magnitude between the calibration star and another standard star used for checking,

$\sigma=\sqrt{\frac{\sum\left(m_{i}-\bar{m}\right)^{2}}{N-1}}$

where $m_{i}=\left(m_{\mathrm{C}}-m_{\mathrm{K}}\right)_{i}$ is the differential magnitude of the calibration star $\mathrm{C}$ and check star $\mathrm{K}$, while $\bar{m}=\overline{m_{\mathrm{C}}-m_{\mathrm{K}}}$ is the differential magnitude averaged over the entire data set, and $N$ is the number of observations on a given night. For each object, the typical rms error is between 0.01 and $0.02 \mathrm{mag}$.

\section{Flux variability}

The results of our monitoring program are listed in Table 2, available in electronic form at the CDS. Column 1 is the object name, Col. 2 is the Julian Date, Col. 3 is the magnitude, Col. 4 is the rms magnitude error, Col. 5 is the filter used, and Col. 6 is the observatory. For each object, the historical photometric results are briefly presented. The overall magnitude variations of each object are investigated. Variations on time scales of days, and on shorter time scales (e.g. hours), in several objects are also reported.

${ }^{1}$ IRAF is distributed by the National Optical Astronomy Observatory, which is operated by the Association of Universities for Research in Astronomy, Inc., under cooperative agreement with the National Science Foundation.

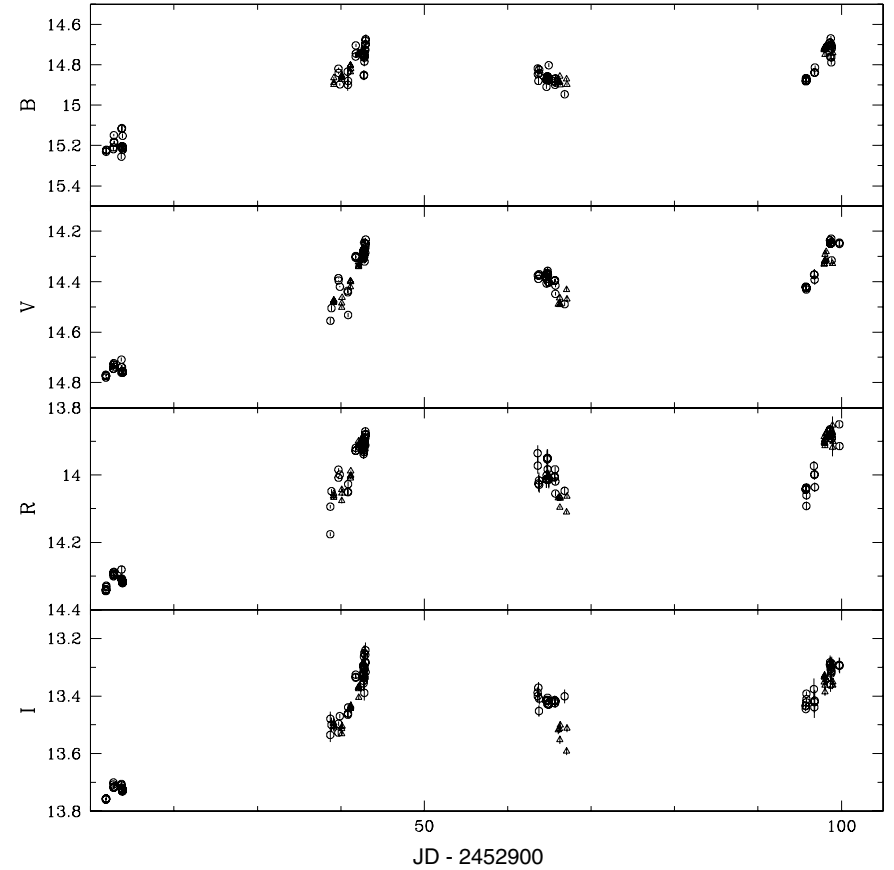

Fig. 1. Light curves of $3 \mathrm{C} 66 \mathrm{~A}$ in the $B, V, R, I$ bands. The circles represent the data from the $1.0 \mathrm{~m} \mathrm{Mt}$. Lemmon telescope, and the triangles are from the $0.6 \mathrm{~m}$ Sobaeksan telescope.

\section{1. $3 C 66 \mathrm{~A}$}

The blazar 3C 66A is a member of the Einstein Slew Survey sample (Elvis et al. 1992). Both GeV (Dingus et al. 1996; Mukherjee et al. 1997) and TeV (Neshpor et al. 1998) $\gamma$-rays have been detected in this highly polarized (Mead et al. 1990) blazar. This source has displayed infrared, optical, ultraviolet, and X-ray variability on different time scales (Ghosh \& Soundararajaperumal 1995; De Diego et al. 1997; Carini et al. 1998). 3C 66A was the target of an extensive multiwavelength Whole Earth Blazar Telescope (WEBT) monitoring campaign from July 2003 through April 2004, involving observations in radio, infrared, optical, X-ray, and very-high-energy $\gamma$-ray bands (Böttcher et al. 2005). At all optical frequencies, a gradual brightening of the source over the course of the campaign was observed, with a maximum at $R \approx 13.4$ on Feb. 18, 2004. Microvariability with flux changes of $\sim 5 \%$ on time scales as short as $\sim 2 \mathrm{~h}$, and several bright flares on time scales of several days, were detected (Böttcher et al. 2005). Our observational data on this source have been included in the WEBT campaign paper (Böttcher et al. 2005).

Our BVRI light curves are shown in Fig. 1. Data obtained from different observatories are represented by different symbols. During our observing runs, the overall magnitude variations were $\Delta B=0.59, \Delta V=0.55, \Delta R=0.50$, and $\Delta I=0.52$ over 88 days. Our observations of this object consist of four sessions: September, October, November, and December 2003. From Fig. 1, it can be seen that the source stays at a relatively low state, $R \sim 14.3$ in the September session. However, 3C 66A brightened to $R \sim 14.0$ in the following three sessions. It can also be seen that the source brightens gradually in the October, and December sessions, but fades in the November session, 


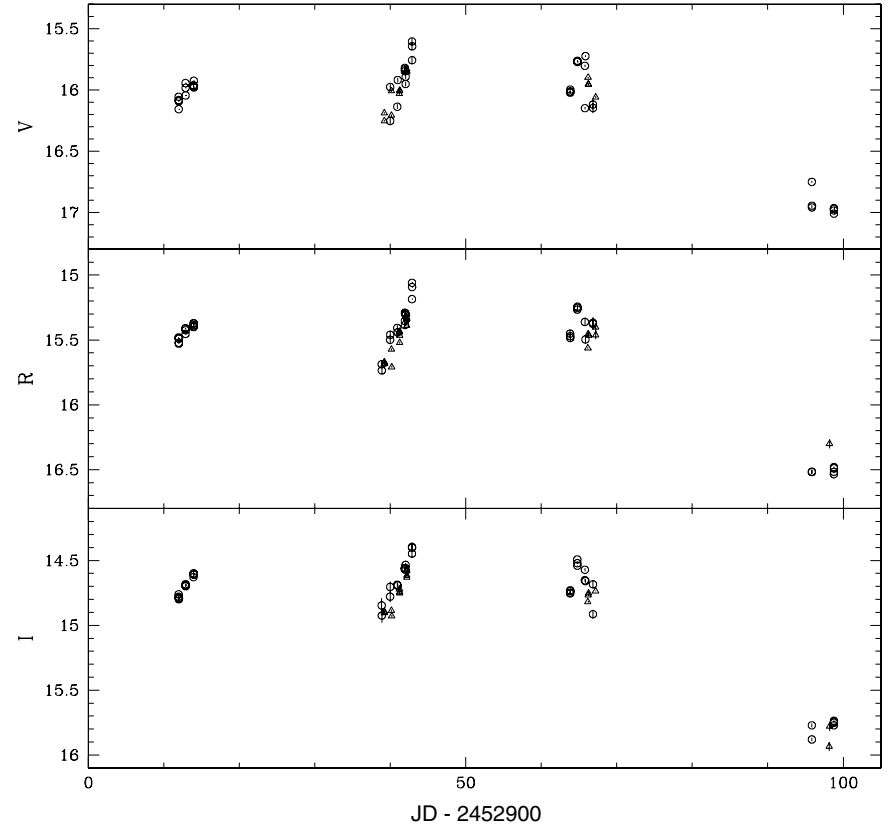

Fig. 2. Light curves of PKS $0420-01$ in the $V, R, I$ bands. The symbols are the same as in Fig. 1.

although there are fluctuations in each observing run. We find that the brightest magnitude of October, $R \sim 13.8$ is same as that of December. The time separation of these two epochs is about 57 days. Short-time-scale variations (e.g. intraday variations) were also searched for during our observing runs, but no significant such variations were found.

\subsection{PKS 0420-01}

This quasar has revealed a strong variability in the optical band (Webb et al. 1988). A noticeable flare was detected in late 1979, when a 1.3 mag increase in 5 days was registered, followed by a 1.7 mag decrease in 23 days (Villata et al. 1997). A strong variability is also evident from the $20 \mathrm{yr}$ light curve (from 1970 to 1990) reported by Smith et al. (1993). Wagner et al. (1995) noticed flux variations with time scales of the order of 1-10 days. The flare of 1992 February-March was the highest optical state observed until then $(R=14.6)$; in that period EGRET registered the highest $\gamma$-ray flux density. Since low fluxes or non-detections at $\gamma$-ray energies correspond to low optical states, a direct correlation between the optical and $\gamma$-ray emission was suggested. The most noticeable variation was a fall of 2.64 mag in 40 days observed from 1995 September 15 to October 25 in the $R$ band (Villata et al. 1997). Microvariability with $\Delta R=0.12 \mathrm{mag}$ in $40 \mathrm{~min}$ was also detected (Villata et al. 1997).

The VRI light curves of PKS 0420-01 are shown in Fig. 2. During our observing runs, the overall magnitude variations were $\Delta V=1.41, \Delta R=1.48$, and $\Delta I=1.54$. The maximum $R=15.06$ happened on October 30, 2003 (JD = 2452942.87 ), and the minimum $R=16.54$ was on December 25, 2003 (JD = 2452 998.76). During the September run, the light curve over three days (September 29, 30, and October 1) shows that the source was near $R \sim 15.4$, and it slightly brightened over this period, but with only about 0.12 mag variation in this band. During the October session, the source gradually brightened from $R=15.74 \pm 0.03$ on October 26 , to $R=15.06 \pm 0.01$ on October 30, which is the maximum over the whole monitoring period. The average brightness during these five days is $R \sim 15.4$, which is same as the average magnitude of the September session. We find that the source also stays at the same brightness level $(R \sim 15.4)$ in the November run, although there are fluctuations from day to day. The $R$ magnitude was $\sim 15.47$ on November 20 , and it brightened to $\sim 15.25$ on November 21. On November 22, the source faded to $R \sim 15.43$, and after that, it stayed at $R \sim 15.4$. However, the source had faded by about 1 mag to $R \sim 16.5$ in the December session.

\section{3. $S 50716+71$}

The BL Lac object S5 0716+71 was included in the S5 cata$\log$ of the Strong Source Survey performed at $4.9 \mathrm{GHz}$ (Kühr et al. 1981). The largest optical database on S5 0716+71 ever published was collected and analyzed in Raiteri et al. (2003), which consists of a total of $4854 U B V R I$ data points obtained from eight observatories from 1994 to 2001. Four major optical outbursts were observed: at the beginning of 1995, in late 1997, at the end of 2000, and in autumn 2001. In particular, an exceptional brightening of 2.3 mag in 9 days was detected in the $R$ band just before the BeppoSAX pointing of October 30, 2000. They found that the long-term trend shown by the optical light curves seems to vary with a characteristic time scale of about 3.3 years. The source has been recently monitored at radio and optical wavelengths by more than 40 telescopes in the northern hemisphere, during a WEBT campaign lasting from September 2003 to June 2004. Nesci et al. (2002) presented a study of the optical intraday variability (IDV) of S5 $0716+71$ over 52 nights. They found typical variation rates of 0.02 mag per hour, and a maximum rising rate of 0.16 mag per hour. S5 $0716+71$ has been observed with INTEGRAL on 2-6 April 2004 (Pian et al. 2005). It was detected with IBIS/ISGRI up to $60 \mathrm{keV}$, with a flux of $\sim 3 \times 10^{-11} \mathrm{erg} \mathrm{s}^{-1} \mathrm{~cm}^{-2}$ in the $30-60 \mathrm{keV}$ interval.

S5 $0716+71$ was the most extensively monitored source in our observations. The observations span about 140 days, occurring in September, October, November, December 2003, and February 2004. The light curves in the Johnson's $B V$ and Cousins' $R I$ bands are shown in Fig. 3, where the different symbols refer to the various telescopes listed in Table 1. It can be seen from Fig. 3 that the source exhibited strong variability, with similar trends (but with slightly different amplitudes) in all bands. The overall magnitude variations were $\Delta B=1.42$, $\Delta V=1.37, \Delta R=1.37, \Delta I=1.36$, where the differences are mainly due to the different temporal samplings. During our observing runs, the maximum $R$-band brightness, $R=12.62$, was recorded on 10 December 2003 (JD $=2452983.8756$ ). We note that a rapid brightness increase was detected during the 4 days of 19-23 November 2003, and which is shown in Fig. 4. The overall variation in the $R$ band was $\Delta R=1.15$, from $R=13.94 \pm 0.01$ on JD $=2452962.916$ to $R=12.79 \pm 0.01$ 


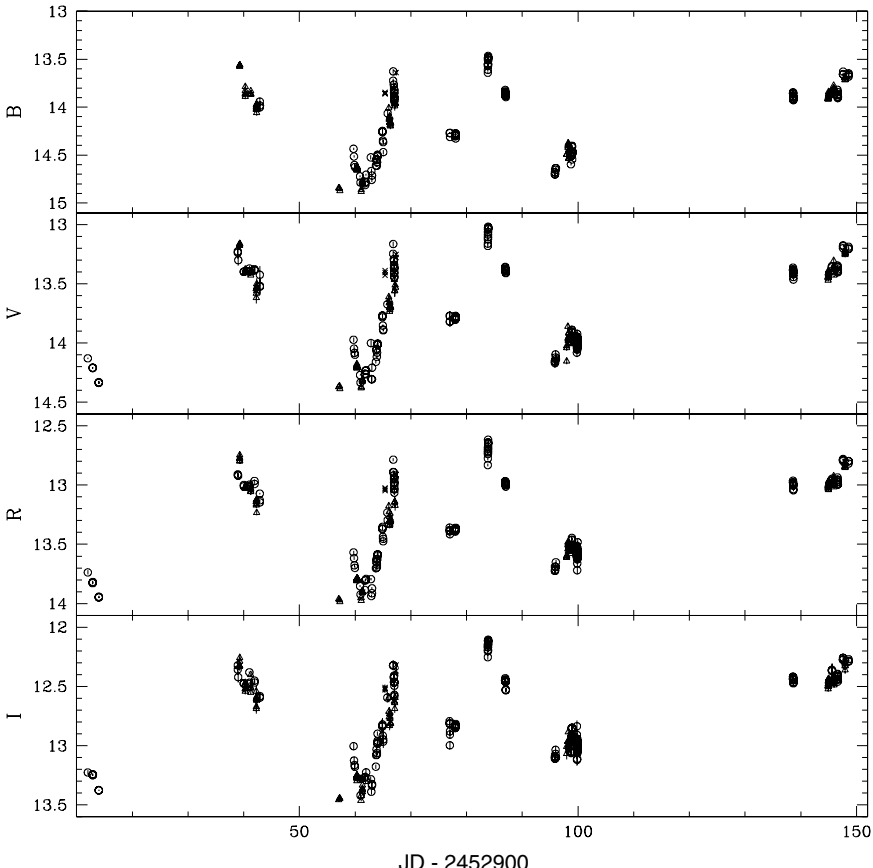

Fig. 3. Light curves of $\mathrm{S} 50716+71$ in the $B, V, R, I$ bands. The circles and triangles are the same as in Fig. 1, and the crosses are the data from the $1.8 \mathrm{~m}$ Bohyunsan telescope.

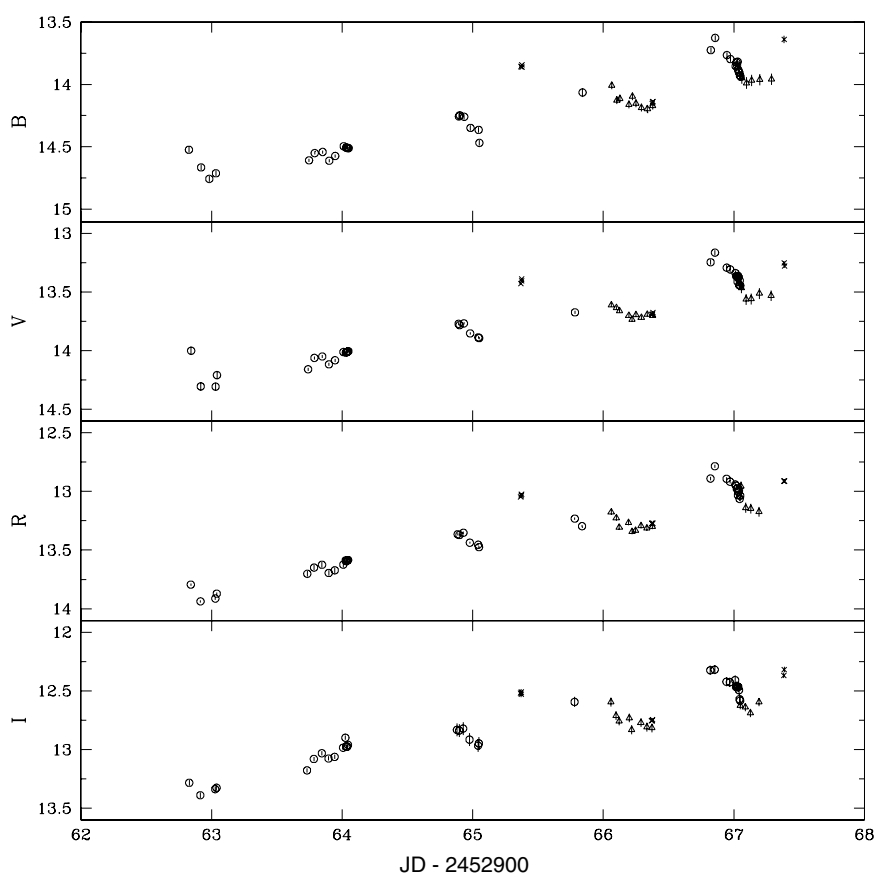

Fig. 4. The variability of S5 0716+71 during 19-23 November 2003, in the $B, V, R, I$ bands. The symbols are the same as in Fig. 3 .

on JD $=2452966.854$, both observed from the Mt. Lemmon Observatory.

Interestingly, we find that the shape of the profile of this rapid brightness increase is significantly linear. We have fitted least-square regression lines to the light curves in the $B V R I$ bands obtained from all three telescopes. The slopes of the best-fit lines are given in Col. 2 of Table 3, together with the regression coefficients for the best fits in Col. 3. It is noted
Table 3. Slopes and regression coefficients $(r)$ of the least-square linear fits to the BVRI magnitude variations of S5 $0716+71$, during 19-23 November 2003.

\begin{tabular}{lcc}
\hline \hline Filters & slope $\pm \sigma$ & $r$ \\
\hline$B$ & $-0.197 \pm 0.013$ & -0.90 \\
$V$ & $-0.194 \pm 0.013$ & -0.90 \\
$R$ & $-0.198 \pm 0.012$ & -0.92 \\
$I$ & $-0.179 \pm 0.012$ & -0.89 \\
\hline
\end{tabular}

that the slopes are essentially the same for the different passbands. The differences in the slopes are always $\leq 1 \sigma$, and are therefore not statistically significant. Our results show that the rapid brightening can be described by linear trends on a magnitude scale (which, therefore, corresponds to exponential intensity variations), and this rapid brightening rate was $\sim 0.2$ mag per day over 4 days. However, it should be noted that this rapid brightening is just a mean behaviour, and may also be due to the lack of data in the gaps.

Rapid variations of the flux of S5 $0716+71$ have been observed on different occasions (e.g. Ghisellini et al. 1997; Sagar et al. 1999; Xie et al. 2004). In our observations, we also searched for intraday variations. Variations, with amplitudes of $>0.15$ mag over a few hours in one night, were found on November 23 and December 10, 2003, and which are shown in Figs. 5 and 6, respectively. Both of them were detected with the Mt. Lemmon Observatory. The lower panels in these figures show the difference between the instrumental magnitude of the two field standard stars. In Fig. 5, it can be seen that the source brightened from $V=13.25 \pm 0.02(R=12.89 \pm 0.01)$ to $V=13.17 \pm 0.02(R=12.79 \pm 0.01)$ within $48 \mathrm{~min}$, and afterwards it faded to $V=13.45 \pm 0.02(R=13.06 \pm 0.01)$ in the remaining observing time $(\sim 4.6 \mathrm{~h})$ for this night. The overall magnitude variations were $\Delta V=0.28 \mathrm{mag}(\Delta R=0.27 \mathrm{mag})$ during this night. The variability parameters $C$ (introduced by Romero et al. 1999; if $C>2.576$, then the source is variable) are 3.64 ( $V$ band), and 5.27 ( $R$ band). The lower panels show that the maximum deviation of the two field standard stars is $\Delta V_{\max }=0.07 \mathrm{mag}$ in the $V$ band, and $\Delta R_{\max }=0.06$ in the $R$ band. In Fig. 6, the source brightened from $V=13.18 \pm 0.01$ $(B=13.64 \pm 0.01)$ to $V=13.02 \pm 0.01(B=13.47 \pm 0.01)$ within about $1.4 \mathrm{~h}$, then faded. The rising rate, of 0.002 mag per minute in both $B$ and $V$ bands, was found over the duration of the first $1.4 \mathrm{~h}$. This is in good agreement with the results of Villata et al. (2000), where there was a monotonic brightness increase from $B=14.13 \pm 0.01$ to $B=13.98 \pm 0.01$ in about $130 \mathrm{~min}$, with the steepest (linear) part having a rising rate of 0.002 mag per minute, and a duration of about $45 \mathrm{~min}$. Moreover, the authors suggested that such a gradient seems to be typical of fast variations, with no evident difference between rise and fall.

\subsection{PKS $0735+17$}

PKS $0735+17$ was first classified as a BL Lac object by Carswell et al. (1974). It is both radio (Kühr et al. 1981) and 

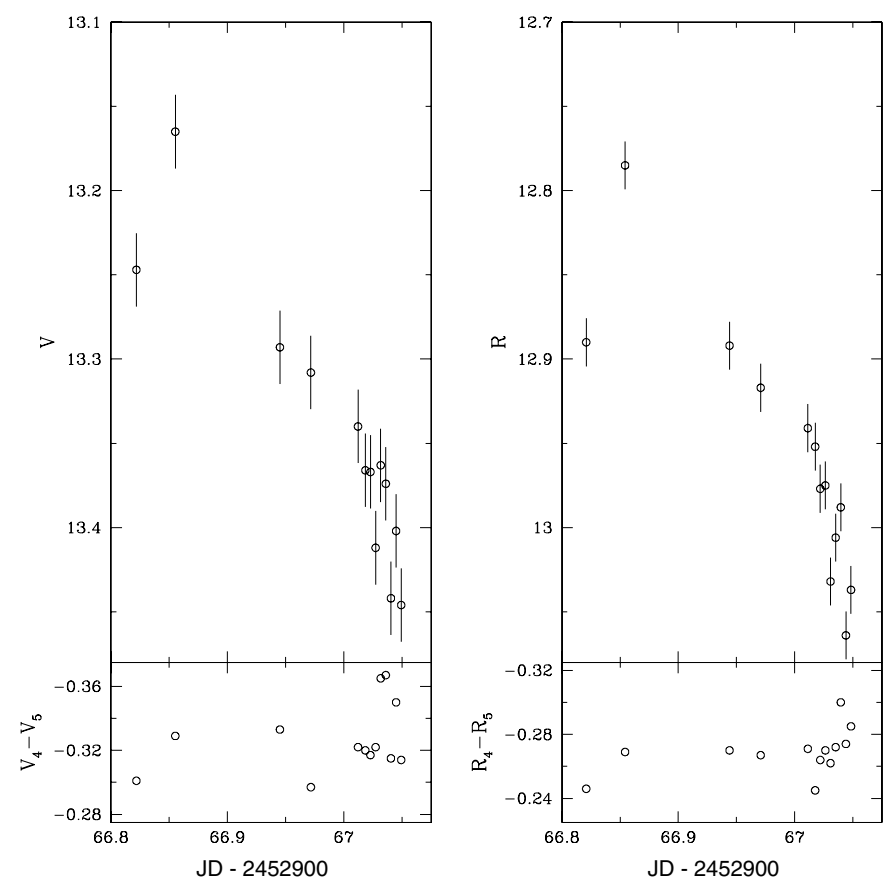

Fig. 5. Intraday variations of S5 $0716+71$ on 23 November 2003, in the $V$ band (left panel) and in the $R$ band (right panel). All data are from the $1.0 \mathrm{~m} \mathrm{Mt}$. Lemmon telescope.
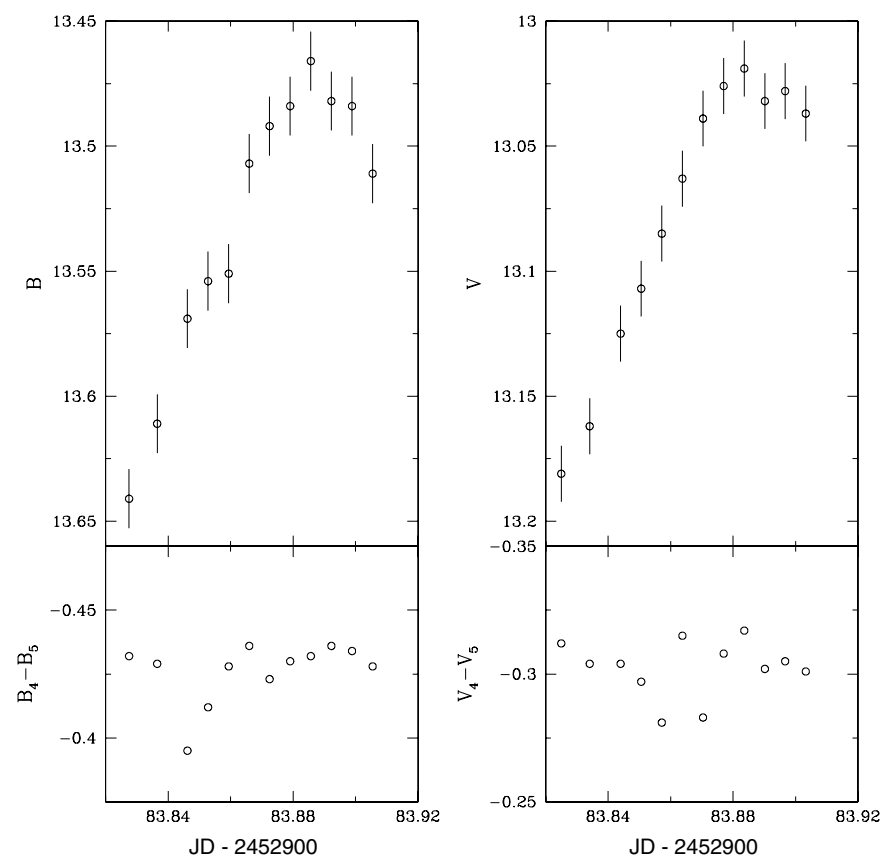

Fig. 6. Intraday variations of S5 $0716+71$ on 10 December 2003, in the $B$ band (left panel) and in the $V$ band (right panel). All data are from the $1.0 \mathrm{~m}$ Mt. Lemmon telescope.

X-ray selected (Elvis et al. 1992). Falomo \& Ulrich (2000) gave a lower limit on the object's redshift, of $z>0.5$, and Xie et al. (2002) have estimated its black hole mass. Tommasi et al. (2001) observed the optical polarimetry of PKS $0735+17$ in 1999 December, and gathered together previous polarimetric data. The source has shown very different levels of polarization percentage in past years, from around $1 \%$ to more than $30 \%$.

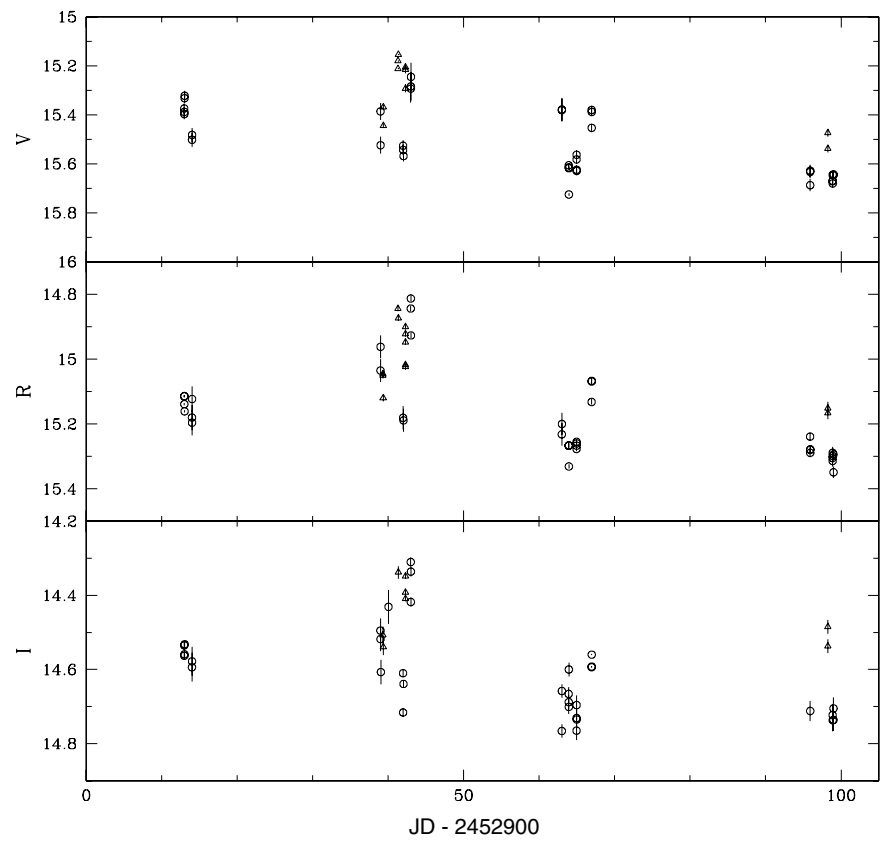

Fig. 7. Light curves of PKS $0735+17$ in the $V, R, I$ bands. The symbols are the same as in Fig. 1.

PKS $0735+17$ is also known as an optical and infrared intraday variable blazar (Massaro et al. 1995; Heidt \& Wagner 1996; Bai et al. 1998). With observational data spanning over $90 \mathrm{yr}$, the long-term periodicity of this blazar has been investigated; its optical variability shows the possibility of a $4.89 \mathrm{yr}$ period (Webb et al. 1988; Smith et al. 1988), and also a $14.2 \mathrm{yr}$ period (Fan et al. 1997).

Our VRI light curves are shown in Fig. 7. During our observing runs, the overall magnitude variations were $\Delta V=0.57$, $\Delta R=0.54$, and $\Delta I=0.46$. From Fig. 7 , it can be seen that this source is variable almost all the time, although the overall variations are not relatively large. This source was relatively stable from September 30 to October 1, 2003. The average $R$ magnitude of PKS $0735+17$ was $15.13 \pm 0.02$ on September 30 , and $15.17 \pm 0.04$ on October 1. From October 26 to October 30, the apparent fluctuations are present in all bands (see Fig. 7). On October 30, the maximum $R$ brightness of whole monitoring period was reached at $14.81 \pm 0.01$ (JD $=2452942.998)$. In the $R$ band, a brightening from $15.26 \pm 0.01$ on November 21 to $15.09 \pm 0.04$ on November 23 was detected. One month later, the object was observed at $R=15.27 \pm 0.02$ on December 22, and brightened to $R=15.16 \pm 0.02$ after two days. Finally, it faded to $R=15.31 \pm 0.02$ on December 25 .

\subsection{OJ 287}

OJ 287 is one of the most extensively observed BL Lac objects. It has been observed for over 100 years, providing a very good historical light curve. In 1972, it reached maximum light, with $V \sim 12$. Pursimo et al. (2000) presented intensive optical, infrared, and radio monitoring of OJ 287, taken between 1993 and 1998. These monitoring results show that the optical and infrared light curves displayed continuous variability on timescales ranging from tens of minutes to years. The 


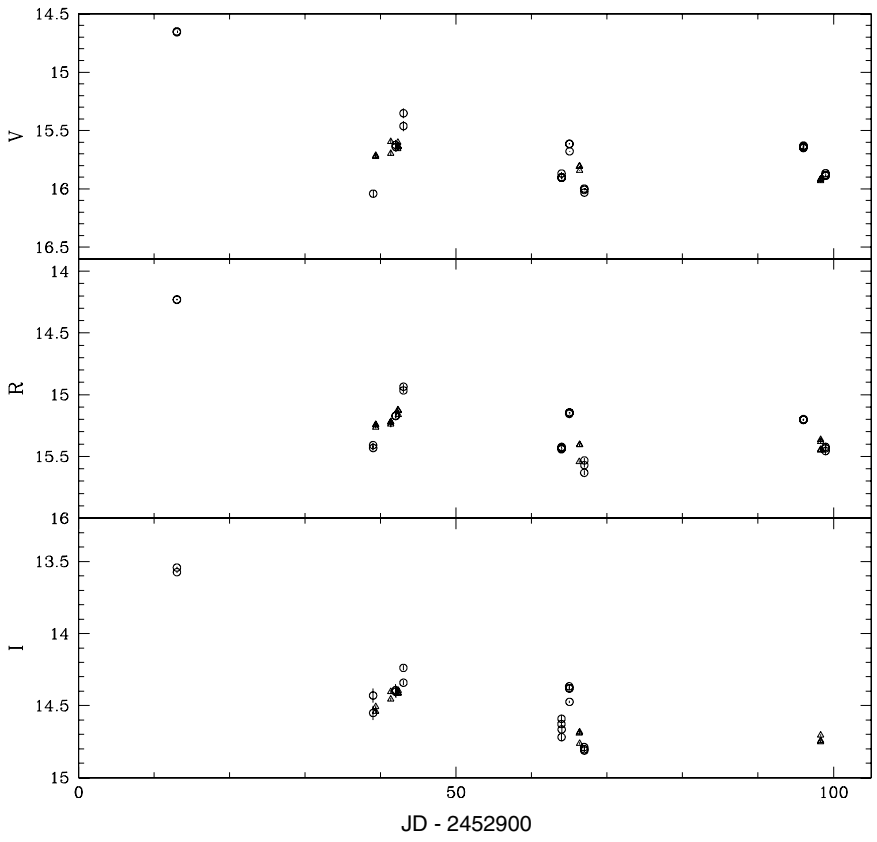

Fig. 8. Light curves of OJ 287 in the $V, R, I$ bands. The symbols are the same as in Fig. 1.

historical light curve shows several outbursts with a recurrence period of about $11.65 \mathrm{yr}$ (Sillanpää et al. 1988a). The $12 \mathrm{yr}$ cycle was discussed in many occasions (e.g. Villata et al. 1998b; Abraham 2000; Katajainen et al. 2000; Valtaoja et al. 2000). Sillanpää et al. (1988a) modeled these periodic outbursts by using a binary supermassive black hole system, and predicted that the next outburst would occur during late 1994. This prediction was confirmed by several studies (Kidger et al. 1995; Sillanpää et al. 1996a,b; Arimoto et al. 1997; Fan et al. 1998a; Jia et al. 1998; Pursimo et al. 2000; Efimov et al. 2002).

Our VRI light curves are shown in Fig. 8. During our observing runs, the overall magnitude variations were $\Delta V=1.39$, $\Delta R=1.40$, and $\Delta I=1.27$. The maximum $R=14.23 \pm 0.01$ occurred on September 30 (JD $=2452913.001)$, whereas the minimum $R=15.63 \pm 0.02$ was on November 23 (JD = 2452 966.982). The source was observed at $R=14.23 \pm 0.01$ $(V=14.65 \pm 0.03, I=13.55 \pm 0.05)$ on September 30, 2003, which was recorded at the Mt. Lemmon Observatory. About one month later, it faded to $R=15.42 \pm 0.02$ on October 26. After that, it gradually brightened, and reached $R=$ $14.95 \pm 0.02$ on October 30 . In November, the source brightness stayed at a similar level as that in October. On November 20, the $R$ magnitude was recorded at $15.43 \pm 0.01$, but it brightened to $15.15 \pm 0.01$ on November 21. Later, the source faded to $15.58 \pm 0.02$ on November 23. In December, a fading from $R=15.20 \pm 0.01$ on December 22, to $R=15.44 \pm 0.03$ on December 25 was detected over three days, at the Mt. Lemmon Observatory.

\section{6. $3 C 345$}

3C 345 was classified as an OVV quasar by Penston \& Cannon (1970). The typical behaviour for this object has been to show about 2 mag outbursts, which occur quite frequently.

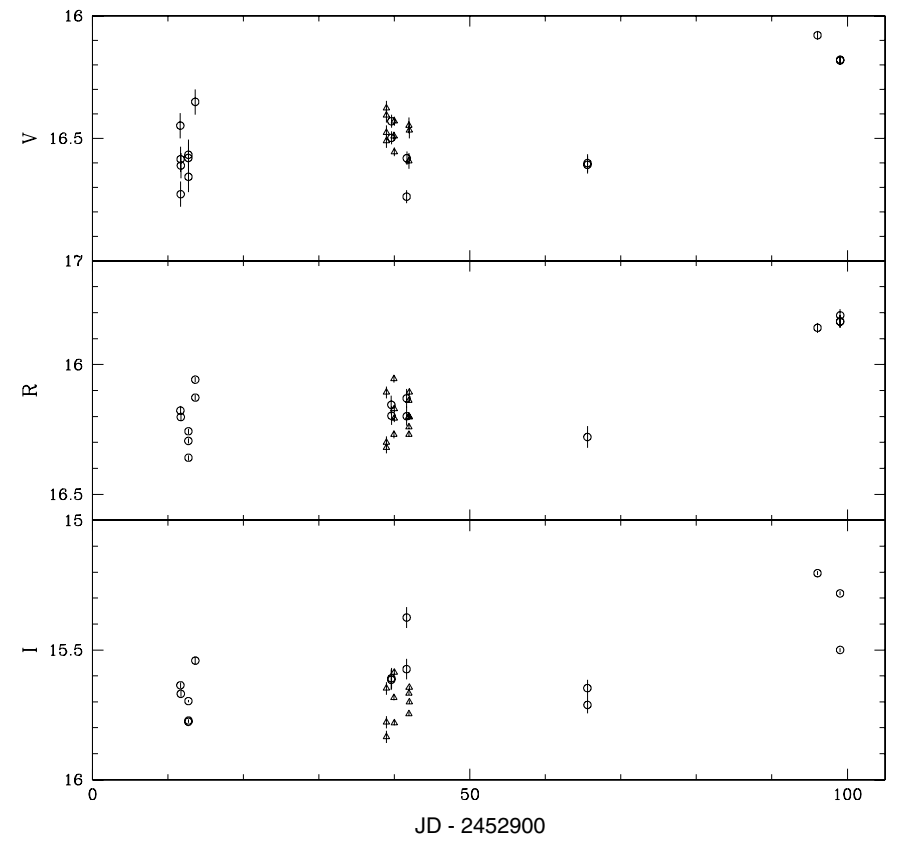

Fig. 9. Light curves of $3 \mathrm{C} 345$ in the $V, R, I$ bands. The symbols are the same as in Fig. 1.

Such large outbursts happened in 1967/1968, 1971/1972, 1982/1983, and 1991/1992 (Schramm et al. 1993). Sillanpää et al. (1988b) measured $V=14.56$ in 1982 , while Schramm et al. (1993) observed the $V$-band brightness near 18 mag in 1989. In 1991/1992, a large 2.5 mag outburst was observed (Schramm et al. 1993). Kidger \& Takalo (1990) observed the historical minimum at $B=18.66$.

The VRI light curves are shown in Fig. 9. During our observing runs, the overall magnitude variations were $\Delta V=0.66$, $\Delta R=0.55$, and $\Delta I=0.63$. The maximum $R$ brightness $R=15.81 \pm 0.02$ was observed on December 25 (JD = 2452 999.055), and the minimum $R=16.36 \pm 0.01$ was on September 30 (JD $=2452912.710)$. Variations with small amplitudes on time scales of days are found in all bands, in September, October, and December 2003.

\subsection{BL Lac}

BL Lac is the prototype of the BL Lac object class of AGNs, and one of the best studied blazars. Shen \& Usher (1970) have investigated its historical light curves, and found a range of variation of $4.2 \mathrm{mag}$ in the $V$ band, and a strong outburst in which BL Lac varied over nearly its entire range (12.4 $\leq$ $B \leq 16.7 \mathrm{mag}$ ) during 400 days. Investigations of its long-term variability have been done by Webb et al. (1988), and Carini et al. (1992), which show that the faintest magnitudes were $B=17.99 \mathrm{mag}$, and $V=16.73 \mathrm{mag}$. Recently, Villata et al. (2004) presented UBVRI light curves obtained by the WEBT from 1994 to 2002, including the last, extended BL Lac 2001 campaign. Their analysis of the colour indices reveals that the flux variability can be interpreted in terms of two components: longer-term variations occurring on a few-day time scales appear as mildly-chromatic events, while a strong bluer-whenbrighter chromatism characterizes very fast (intraday) flares. 


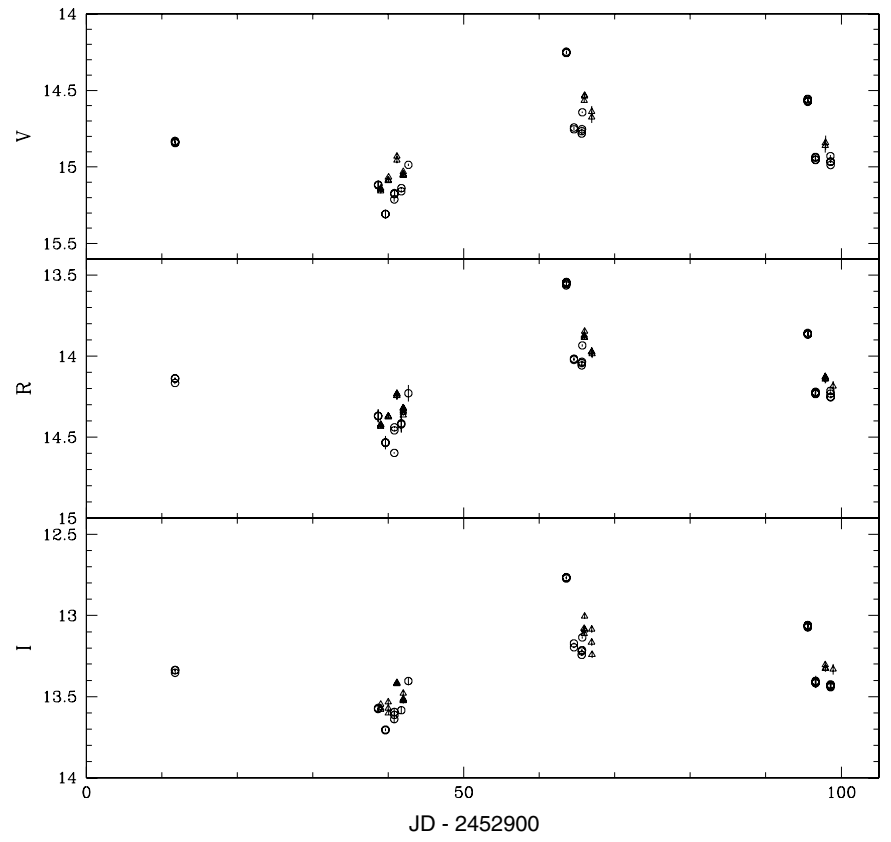

Fig. 10. Light curves of BL Lac in the $V, R, I$ bands. The symbols are the same as in Fig. 1.

They suggested that Doppler factor variations on a "convex" spectrum could be the mechanism accounting for both the longterm variations, and their slight chromatism.

Our VRI light curves are shown in Fig. 10. During our observing runs, the overall magnitude variations were $\Delta V=1.06$, $\Delta R=1.05$, and $\Delta I=0.94$. The maximum $R$-band brightness was recorded at $R=13.55 \pm 0.01$ on November 20 $(\mathrm{JD}=2452963.580)$, whereas the minimum was detected at $R=14.60 \pm 0.01$ on October 28 (JD = 2452940.784$)$. BL Lac was observed at $R \sim 14.15 \pm 0.01$ on September 29. It was slightly fainter in late October. On October 26, the $R$ magnitude was $R=14.37 \pm 0.04$. It became $R=14.23 \pm 0.05$ after four days, during which small amplitude fluctuations $(\leq 0.2 \mathrm{mag})$ were detected from day to day. In the November run, BL Lac first brightened to $R=13.55 \pm 0.01$ on November 20 (JD 2452963.580 ), then it faded to $R=14.02 \pm 0.01$ in one day. On November 22, a brightening of $\sim 0.10 \mathrm{mag}$ in the $R$ band ( $0.11 \mathrm{mag}$ in the $V$ band, and $0.08 \mathrm{mag}$ in the $I$ band), from $R=14.03 \pm 0.01$ at $\mathrm{JD}=2452965.648$ to $R=13.93 \pm 0.01$ at JD $=2452965.696$, was detected in about $69 \mathrm{~min}$. The $R$ magnitude was recorded at $13.86 \pm 0.01$ on December 22 , but it dropped to $14.23 \pm 0.01$ after one day. After that, it brightened slightly to $14.14 \pm 0.02$ on December 24 . Finally, it reached $14.24 \pm 0.02$ on December 25 .

\section{8. $3 C 454.3$}

3C 454.3 is a GeV $\gamma$-ray source, and one of the few blazars that have been detected by COMPTEL (Blom et al. 1995). The light curve of 3C 454.3 in the $B$ band from 1966 to 1979 is given by Lloyd (1984). Data in the $B$ band from 1971 to 1985 are reported by Webb et al. (1988), and a flare with a variation of $1.28 \mathrm{mag}$ in 63 days was reported in autumn 1979. From 1986 November to 1987 January, the average

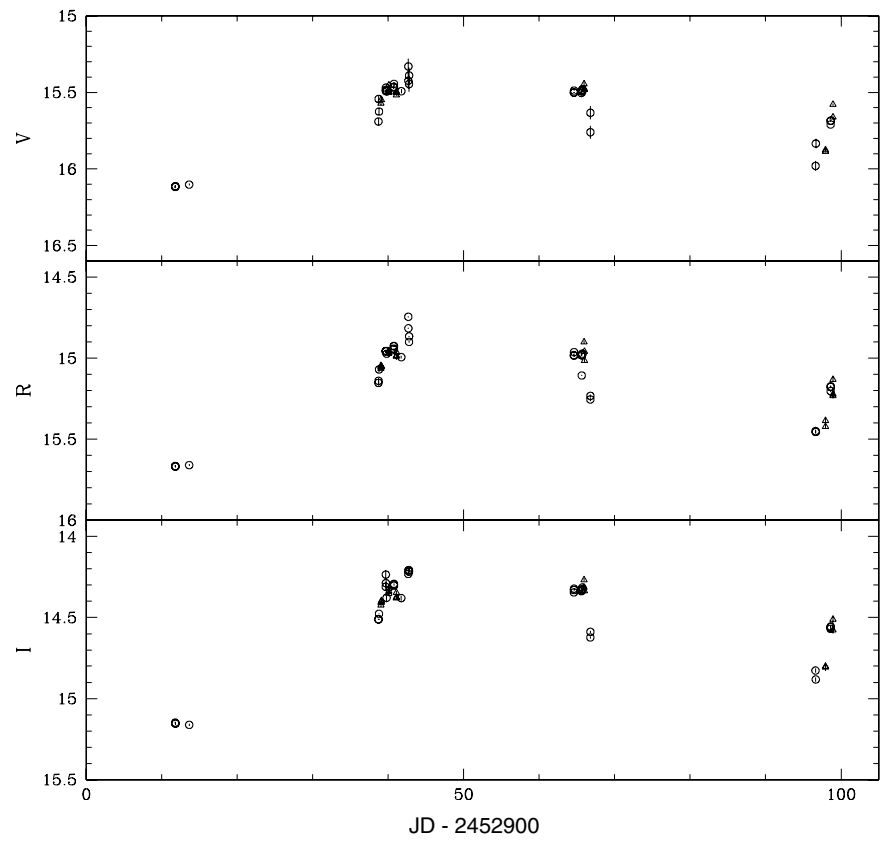

Fig. 11. Light curves of $3 \mathrm{C} 454.3$ in the $V, R, I$ bands. The symbols are the same as in Fig. 1.

$B$ magnitude of the source was 16.56 , with a variation of 0.92 mag over 44 days (Corso et al. 1988). The results of the monitoring program of Villata et al. (1997) show that a fast variation of $0.27 \mathrm{mag}$ in $2.6 \mathrm{~h}$ occurred in the $R$ band; and that in any band this source has presented only small, short-term variations, within a total range of less than 0.4 mag (Villata et al. 1997). The $B V R$ light curves of 3C 454.3 from November 1994 to December 1997, were presented by Villata et al. (2001).

The VRI light curves are shown in Fig. 11. During our observing runs, the overall magnitude variations were $\Delta V=0.79$, $\Delta R=0.93$, and $\Delta I=0.95$. The maximum $R$ magnitude $14.75 \pm 0.01$ was found on October 30 (JD = 2452942.662$)$, while the minimum $15.67 \pm 0.01$ was on September 29 (JD = 2452 911.810). On September 29 and October 1, the source was stable at $R=15.67 \pm 0.01(V=16.11 \pm 0.01, I=$ $15.15 \pm 0.01)$. The object became brighter in late October. It was detected at $R \sim 15.1$ on October 26, and it gradually brightened over the following four days, until it reached $R \sim 14.8$ on October 30. On November 21 and 22, it was stable at $R \sim 15.0$, but faded to $R \sim 15.2$ on November 23. In December, it started at $R=15.45 \pm 0.01$ on December 23, then brightened slightly on December 24. Finally, an $R$ magnitude of $15.13 \pm 0.02$ was reached on December 25, as recorded by the Sobaeksan Observatory.

\section{Spectral variability}

In this section, we investigate the relationship between the spectral changes and the flux variations. The plots of the colour indices $V-I$ and $R$ magnitudes for each object are given in Fig. 12. In most cases, the colour indices were calculated by coupling the data taken by the same instrument within $10 \mathrm{~min}$ intervals. In Table 4, we report the principal results of our 


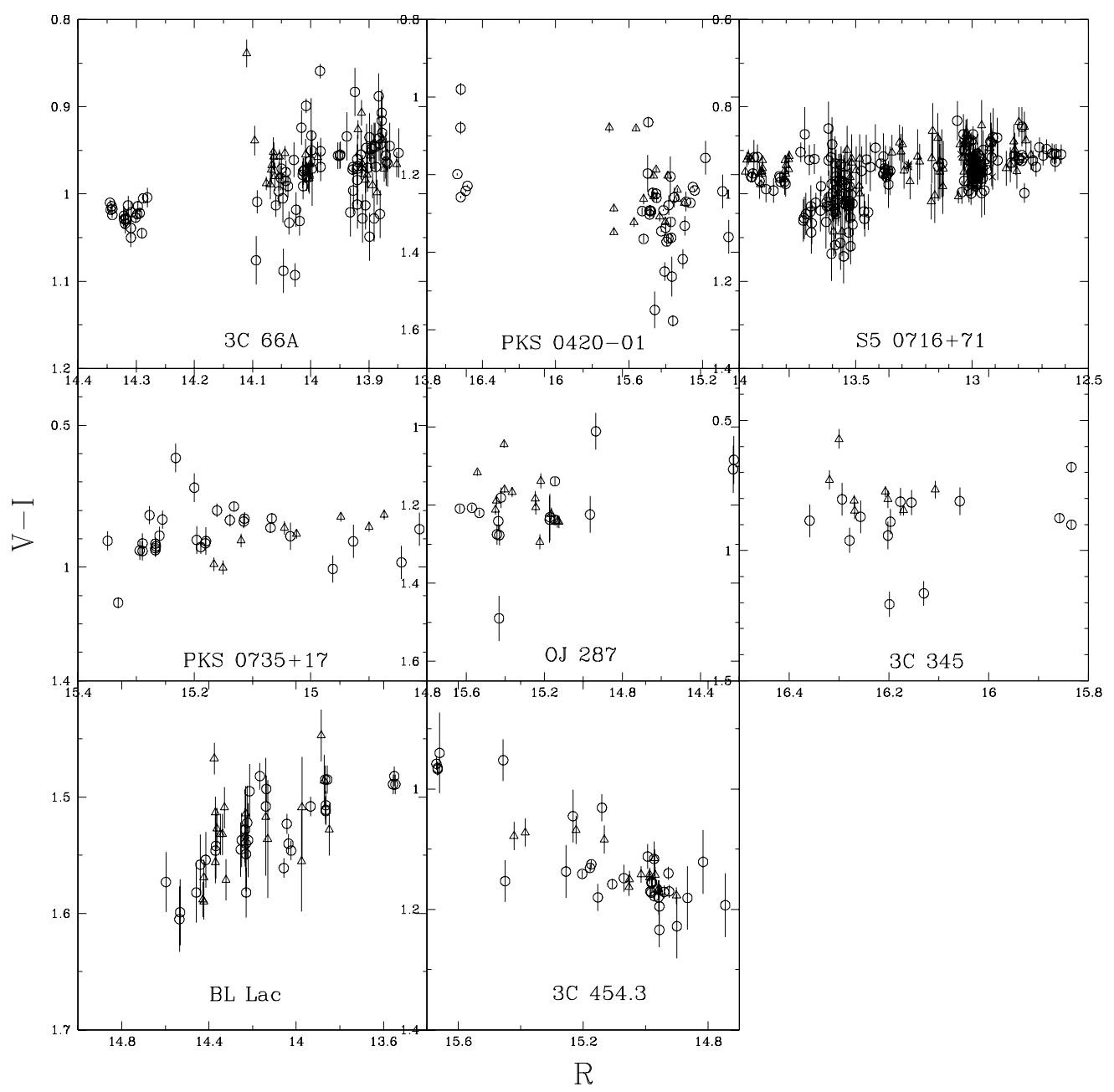

Fig. 12. The $V-I$ colour indices versus $R$ magnitudes for the eight blazars. The rms colour magnitude errors are indicated by the vertical lines. The symbols are the same as in Fig. 3.

Table 4. The correlations between $V-I$ colour index and $R$ magnitude, for the eight blazar objects.

\begin{tabular}{lcccc}
\hline \hline Source & $\Delta R$ & $N$ & $r$ & Probs. \\
\hline 3C 66A & 0.50 & 120 & 0.511 & $7.34 \times 10^{-10}$ \\
PKS 0420-01 & 1.48 & 55 & -0.388 & $2.62 \times 10^{-3}$ \\
S5 0716+71 & 1.37 & 271 & 0.438 & $1.11 \times 10^{-14}$ \\
PKS 0735+17 & 0.54 & 38 & 0.111 & $4.97 \times 10^{-1}$ \\
OJ 287 & 1.40 & 29 & 0.354 & $4.99 \times 10^{-2}$ \\
3C 345 & 0.55 & 22 & -0.009 & $9.66 \times 10^{-1}$ \\
BL Lac & 1.05 & 53 & 0.657 & $1.33 \times 10^{-8}$ \\
3C 454.3 & 0.93 & 48 & -0.837 & $1.07 \times 10^{-16}$ \\
\hline
\end{tabular}

research. Column 1 gives the object name, Col. 2 the variability range in $R$ magnitude, Col. 3 the number of data points used to calculate the colour indices $V-I$, Col. 4 the linear Pearson correlation coefficient between the $V-I$ and $R$ magnitudes, and Col. 5 the probability that no correlation is present.

From Fig. 12, it can be seen that there are no uniform trends of the colour index changes with the source brightnesses, and the significance of the dependence of the variation of the colour index on the source brightness (e.g. as shown by the correlation coefficient $r$ ) is not related with the overall variability range in $R$ magnitude, nor with the number of data points. Interestingly, we find that the colour indices of two out of three FSRQs, PKS 0420-01, and 3C 454.3, tend to be redder when the sources are brighter. Especially, we find a significant anti-correlation between the $V-I$ colour index and $R$ magnitude for $3 C$ 454.3. The linear Pearson correlation coefficient is found to be $r=-0.837$, with a probability of $1.07 \times 10^{-16}$ that no correlation is present. The anti-correlation in PKS 0420-01 is relatively weak, but still significant; however, no significant correlation is found for FSRQ 3C 345. These anti-correlations imply that the spectrum becomes redder (steeper) when the source is brighter. However, this result is opposite to the common colour change tendency in blazars, that the spectrum becomes flatter when the source brightens (Ghisellini et al. 1997; Ghosh et al. 2000; Clements \& Carini 2001; Raiteri et al. 2001; Villata et al. 2002). In contrast to our FSRQs, we find that all our BL Lac objects tend to be bluer (flatter) when they are brighter. We find significant positive correlations between the colour indices and $R$ magnitudes for 3C 66A, S5 0716+71, and BL Lac, with linear Pearson correlation coefficients of $r=0.511$, $r=0.438$, and $r=0.657$, respectively, at $\gg 99.99$ per cent 
confidence; while only weak correlations are found in PKS 0735+17 and OJ 287, at very low confidence levels. The different trends of the colour index variations with brightness in FSRQs and BL Lac objects are somewhat unusual. However, this may indicate that there are different physical conditions in these two populations, although all of them were selected as being red blazars in the present work. Nevertheless, it should be noted that our results are based on limited data which span only a short period (about three months). Certainly, more observations are needed to reinvestigate the relationship between the colour index variations with the source brightnesses for our sample. In particular, it would be necessary to confirm and/or check the possible anti-correlation for FSRQs with more observations, and then to further explore the observed differences between FSRQs and BL Lac objects.

As shown in Sect. 3.3, variations on different time scales have been found for the most extensively monitored source in our campaign: S5 0716+71. We then explored the correlation between the colour indices and the source brightness on different time scales. It can be seen from Table 4 that there is a significant correlation with linear Pearson correlation coefficient $r=0.438$, using the whole data set, which spans about 140 days. During the rapid brightening over the 4 days of 19-23 November 2003 (Fig. 4), we find a significant correlation with linear Pearson correlation coefficient $r=0.656$, which suggests that the trend of flatter spectra when brighter also holds for variations on time scales of days. This spectral flattening with increasing brightness was also recognized by Villata et al. (2000) in the 72-h optical light curves obtained for S5 0716+71, during the WEBT campaign of February 1999. However, we do not find any significant correlations between the colour index and brightness during the intraday variations, either on 23 November 2003 (Fig. 5) or on 10 December 2003 (Fig. 6). Ghisellini et al. (1997) detected a spectral flattening when the flux is higher during rapid flares; however, no correlation between spectral index and brightness level was found in the long-term trend. A curved trajectory of a relativistic emitting blob, or very rapid electron injection and cooling processes, are proposed by the authors to interpret the fast variations. Recently, using a large database, Raiteri et al. (2003) found that the optical colour indices are only weakly correlated with brightness, and that a clear spectral steepening trend was observed during at least one long-lasting dimming phase (JD 2451 545-2 451 630). However, different spectral behaviours were found on shorter time scales.

In the case of $3 \mathrm{C} 66 \mathrm{~A}$, the trend of flatter spectra when brighter is consistent with the recent results of Vagnetti et al. (2003), who have found a consistent trend of $B-R$ hardening with increasing $B$ band flux, independent of the actual flux value. Ghosh et al. (2000) found that the spectral index of the source, between $V$ and $R$ bands, flattened when the source brightness in $V$ remained almost constant, while decreasing in $R$, between 1997 December 15 and 1998 January 2. Also, between 1998 January 19 and 30, the source brightness decreased, while the $V-R$ colour index reddened, and that this correlated well with the variation of the source brightness. However, from an analysis of a much larger data set of an extensive multiwavelength WEBT monitoring campaign from July 2003 through
April 2004, Böttcher et al. (2005) found that there is a weak indication of a positive hardness-intensity correlation at low flux states with $R \gtrsim 14.0$, whereas no correlation is apparent at higher flux levels. These authors claimed that this might be a consequence of the fact that the $B-R$ hardness actually peaks during the rising phase of individual outbursts.

\section{Discussion}

It is notable that our FSRQs in general, and 3C 454.3 in particular, became redder when brighter (see Fig. 12). In other words, the spectrum became steeper when the object was brighter, and flatter when fainter. This behaviour is opposite to the common trends for blazars, that they become bluer when they brighten (Ghisellini et al. 1997; Fan et al. 1998b; Massaro et al. 1998; Fan \& Lin 1999; Ghosh et al. 2000; Clements \& Carini 2001; Raiteri et al. 2001; Villata et al. 2002). It has been found by different investigators that the amplitude of the variations is systematically larger at higher frequencies, which suggests that the spectrum becomes steeper when the source brightness decreases, and flatter when it increases (Racine 1970; Gear et al. 1986; Ghisellini et al. 1997; Maesano et al. 1997; Massaro et al. 1998). Recent investigations on spectral variability also show this general trend (D'Amicis et al. 2002; Vagnetti et al. 2003; Fiorucci et al. 2004). From our investigations, the variations of the colour indices of our BL Lac objects indeed follow this trend, e.g. 3C 66A, S5 0716+71, and BL Lac. This common phenomenon may be explained in different ways (Fiorucci et al. 2004). It may indicate the presence of two components that contribute to the overall emission in the optical region, one variable (with a flatter slope $\alpha_{\mathrm{var}}$, where $f_{v} \propto v^{-\alpha}$ ), and the other stable (with $\alpha_{\text {const }}>\alpha_{\text {var }}$ ). It is also possible to explain it with a one-component synchrotron model: the more intense the energy release, the higher the particle's energy (Fiorucci et al. 2004). Moreover, it could also be explained if the luminosity increase was due to the injection of fresh electrons, with an energy distribution harder than that of the previous, partially cooled ones (e.g. Kirk et al. 1998; Mastichiadis \& Kirk 2002). In addition, it could be due to a Doppler factor variations on a spectrum slightly deviating from a power law, e.g. Doppler factor variations on a "convex" spectrum (Villata et al. 2004). It may also be possible that more than one mechanism are at work, as pointed out by Villata et al. (2004), the variability observed in the optical curves of BL Lac can be interpreted in terms of two components: a "mild-chromatic" longer-term component and a "strongly-chromatic" shorter-term one, which can be likely due to Doppler factor variations on a "convex" spectrum and intrinsic phenomena, such as particle acceleration from shock-in-jet events (e.g. Mastichiadis \& Kirk 2002), respectively.

However, some evidence that the amplitudes of variations are not systematically larger at high frequencies has been found on several occasions (see, for example: Malkan \& Moore 1986; Brown et al. 1989; Massaro et al. 1998; Ghosh et al. 2000; Clements et al. 2003; Ramírez et al. 2004). Based on their results, Ghosh et al. (2000) suggested that it may not be correct to generalize that the amplitude of the variation in blazars is systematically larger at higher frequency. In particular, they found 
a reddening (i.e. spectral steepening) in their optical observations of the BL Lac object PKS 0735+17. However, our analysis of this source shows that there is a tendency of blueing (i.e. spectral flattening) as the brightness increases, although the correlation between the colour indices and brightnesses is rather weak (see Fig. 12 and Table 4). Moreover, Ghosh et al. (2000) found that the spectral slope of AO 0235+164 remained almost constant when its brightness increased. They concluded that these characteristics cannot be described simply by energy losses in a pure synchrotron mechanism scenario.

The trend that FSRQ PKS 0736+017 was redder when brighter was noted by Clements et al. (2003). They concluded that the phenomenon appeared to be more related to the nature of the variation, than to the host galaxy or the source brightness. Recently, Ramírez et al. (2004) detected the same peculiar tendency to redden with increased brightness in PKS 0736+017, throughout their observations. In addition, the analysis of their data, and of the data reported by Clements et al. (2003), suggested two varying modes. At low flux levels, small changes in flux correspond to large changes in the spectral slope, while much less pronounced spectral changes correspond to a high brightness state. In both cases, the object reddens when it brightens.

FSRQs usually show strong emission lines, and a thermal contribution that may be comparable to the synchrotron emission in the optical spectral region. Their optical emission is contaminated by thermal emission from the accretion disk and the surrounding regions. Of particular relevance is the presence of the so-called "blue bump", or "UV bump", which flattens the spectral slope in the optical region. Since the thermal contribution is larger in the blue region, the composite spectrum would be flatter than the non-thermal component. Then, when the object is brightening, the non-thermal component has a more dominant contribution to the total flux, and the composite spectrum steepens. This scenario has been used to qualitatively explain the low flux level data of PKS 0736+017 (Ramírez et al. 2004). It can also be applied to explain the general trends of steeper spectra when brighter in our FSRQs.

Contrary to the large majority of AGNs, which are characterized by optical spectra with prominent emission lines, BL Lac objects have quasi-featureless spectra. In fact, by the definition of this class of AGNs, the line equivalent widths should be very small. As originally proposed by Blandford \& Rees (1978), the weakness of the spectral lines is most probably due to the fact that the underlying non-thermal continuum is boosted by the relativistic beaming of a jet pointing in the observer's direction. In addition, the central ionizing luminosity of BL Lac objects may be relatively too weak to produce prominent emission lines. Consequently, the thermal contribution is rather small in the optical spectral region, as compared to the synchrotron emission, even when the source is in a low state. In actuality, Vagnetti et al. (2003) found that BL Lac objects and quasars are clearly segregated in the $\alpha-\beta$ plane (see their Fig. 3), where $\alpha$ is the average spectral slope, and $\beta$ is a spectral variability index. The lower average spectral index of their eight BL Lac objects indicates the absence, or lower relative weight, of the thermal blue bump component. The authors further proposed that the segregation in the $\alpha-\beta$ plane is a consequence of the different emission mechanisms in the optical band: synchrotron in the case of BL Lac objects, and thermal hot spots on the accretion disk in the case of QSOs. They found that a simple model representing the variability of a synchrotron component could explain the spectral changes of their BL Lac objects. Our BL Lac objects were selected to be Low-energy-peaked BL Lacs (LBLs); thus, we expect to observe them in the descending part of the spectral power distribution, blueward of the peak frequency. The strong variability of BL Lac objects is generally attributed to the synchrotron emission. It is thus not surprising that they generally follow the common trend for blazars, i.e. bluer when brighter, as we observed for our BL Lac objects.

Although the thermal contribution may in general be relatively large in the optical spectral region in FSRQs, the amount of this contribution can be quite different from source to source. It is conceivable that the thermal contribution is larger in $3 \mathrm{C} 454.3$, as compared to $3 \mathrm{C} 345$, in which only a weak correlation is found. It might be interesting to estimate the thermal contribution in these sources, to better understand the tendency of the colour index to vary with brightness. Nevertheless, it is not easy to distinguish the thermal from the non-thermal components using optical data alone. It is essential to obtain simultaneous observations completely covering at least the near-IR-to-optical part of the spectrum, to better understand the spectral variability of FSRQs and/or blazars. There is no doubt that more observations of blazars in general, and those blazars that show reddening as they brighten in particular, are needed to investigate the relationship between colour index and/or spectral slope and brightness in more detail.

\section{Summary}

We present the results of our monitoring of the flux variability of eight red blazars from 2003 September to 2004 February. The main results can be summarized as follows:

1. All sources showed strong variabilities with amplitudes $>0.5$ mag during our 3-4 month monitoring period. Variations with amplitudes of over 1 mag are found in four sources. In the extreme cases, i.e. PKS 0420-01 and OJ 287, large variations with amplitudes of over 1.4 mag were observed.

2. Intraday variations were found in S5 $0716+71$. Variations with amplitudes $>0.15$ mag were detected on November 23 and December 10, 2003. A rapid rising rate of $0.002 \mathrm{mag}$ per min was found on December 10, 2003. We found a mean behaviour of a rapid linear brightening with a rising rate of $\sim 0.2$ mag per day over 4 days, during November 19-23, 2003.

3. We found that two out of three FSRQs tend to be redder when the sources are brighter; and, conversely, all our BL Lac objects tend to be bluer. There is a significant anticorrelation between the $V-R$ colour index and $R$ magnitude for $3 \mathrm{C} 454.3$, which is opposite to the common colour change tendency in blazars. For our 3 BL Lac objects, 3C 66A, S5 0716+71, and BL Lac, there are strong positive correlations, while only very weak correlations were 
found for the remaining 2 BL Lac objects, PKS 0735+17, and OJ 287.

4. We propose that the different relative contributions of the thermal versus non-thermal radiation to the optical emission may be responsible for the different trends of the colour index with brightness in FSRQs and BL Lac objects.

Acknowledgements. We are thankful to Dr. Jinming Bai (Yunnan Observatory, Chinese Academy of Sciences, China) for stimulating discussions that helped to improve this paper. We thank the anonymous referee for insightful comments and constructive suggestions. We owe great thanks to the observatory staff who supported our blazar monitoring campaign: Bohyunsan Optical Astronomy Observatory, Sobaeksan Optical Astronomy Observatory, and Mt. Lemmon Observatory. This work was partly supported by the National Natural Science Foundation of China (grants 10103003, 10373019 and 10543002) and the Korea Science and Engineering Foundation (grant F01-2005-000-10209-0).

\section{References}

Abraham, Z. 2000, A\&A, 355, 915

Arimoto, J., Sadakane, K., Honda, S., \& Tanabe, K. 1997, PASP, 109, 300

Bai, J. M., \& Lee, M. G. 2001, ApJ, 549, L173

Bai, J. M., Xie, G. Z., Li, K. H., \& Liu, W. W. 1998, A\&AS, 132, 83

Blandford, R. D., \& Rees, M. J. 1978, in Pittsburgh Conference on BL Lac Objects, Pittsburgh, PA, April 24-26, 1978, ed. A. M. Wolfe (Univ. of Pittsburgh Press), 328

Blom, J. J., Bloemen, H., Bennett, K., et al. 1995, A\&A, 295, 330

Böttcher, M., Harvey, J., Joshi, M., et al. 2005, ApJ, 631, 169

Brown, L. M., Robson, E. I., Gear, W. K., \& Smith, M. G. 1989, ApJ, 340,150

Carini, M. T., Miller, H. R., Noble, J. C., \& Goodrich, B. D. 1992, AJ, 104,15

Carini, M. T., Noble, J. C., \& Miller, H. R. 1998, in BL Lac Phenomenon (San Francisco: ASP), 85

Carswell, R. F., Strittmatter, P. A., Williams, R. E., Kinman, T. D., \& Serkowski, K. 1974, ApJ, 190, L101

Catanese, M., \& Weeks, T. 1999, PASP, 111, 1193

Clements, S. D., \& Carini, M. T. 2001, AJ, 121, 90

Clements, S. D., Jenks, A., \& Torres, Y. 2003, AJ, 126, 37

Collmar, W., Reimer, O., Bennett, K., et al. 2000, A\&A, 354, 513

Corso, G. J., Harris, R., Ringwald, F., Shultz, J., \& Mikolajczyk, D. 1988, PASP, 100, 70

D'Amicis, R., Nesci, R., Massaro, E., et al. 2002, PASA, 19, 111

De Diego, J. A., Kidger, M. R., González-Pérez, J. N., \& Lehto, H. J. 1997, A\&A, 318, 331

Dingus, B. L., Bertsch, D. L., Digel, S. W., et al. 1996, ApJ, 467, 589

Efimov, Y. S., Shakhovskoy, N. M., Takalo, L. O., \& Sillanpää, A. 2002, A\&A, 381, 408

Elvis, M., Plummer, D., Schachter, J., \& Fabbiano, G. 1992, ApJS, 80, 257

Falomo, R., \& Ulrich, M. H. 2000, A\&A, 357, 91

Fan, J. H., \& Lin, R. G. 1999, ApJS, 121, 131

Fan, J. H., Xie, G. Z., Lin, R. G., et al. 1997, A\&AS, 125, 525

Fan, J. H., Adam, G., Xie, G. Z., et al. 1998a, A\&AS, 133, 163

Fan, J. H., Xie, G. Z., Pecontal, E., Pecontal, A., \& Copin, Y. 1998b, ApJ, 507, 173
Fiorucci, M., \& Tosti, G. 1996, A\&AS, 116, 403

Fiorucci, M., Tosti, G., \& Rizzi, N. 1998, PASP, 110, 105

Fiorucci, M., Ciprini, S., \& Tosti, G. 2004, A\&A, 419, 25

Fossati, G., Maraschi, L., Celotti, A., Comastri, A., \& Ghisellini, G. 1998, MNRAS, 299, 433

Gear, W. K., Robson, E. I., \& Brown, L. M. J. 1986, Nature, 324, 546

Ghisellini, G., Villata, M., Raiteri, C. M., et al. 1997, A\&A, 327, 61

Ghosh, K. K., \& Soundararajaperumal, S. 1995, ApJS, 100, 37

Ghosh, K. K., Ramsey, B. D., Sadun, A. C., \& Soundararajaperumal, S. 2000, ApJS, 127, 11

González-Pérez, J. N., Kidger, M. R., \& Martín-Luis, F. 2001, AJ, 122, 2055

Han, W., Mack, P., Lee, C.-U., et al. 2005, PASJ, 57, 821

Hartman, R. C., Bertsch, D. L., Bloom, S. D., et al. 1999, ApJS, 123, 79

Hartman, R. C., Böttcher, M., Aldering, G., et al. 2001a, ApJ, 553, 683

Hartman, R. C., Villata, M., Balonek, T. J., et al. 2001b, ApJ, 558, 583

Heidt, J., \& Wagner, S. J. 1996, A\&A, 305, 42

Jia, G. B., Cen, X. F., Ma, H. Y., \& Wang, J. C. 1998, A\&AS, 129, 569

Katajainen, S., Takalo, L. O., Sillanpää, A., et al. 2000, A\&AS, 143, 357

Kidger, M. R., \& Takalo, L. O. 1990, A\&A, 239, L9

Kidger, M. R., González-Pérez, J. N., de Diego, J. A., et al. 1995, A\&AS, 113, 431

Kirk, J. G., Rieger, F. M., \& Mastichiadis, A. 1998, A\&A, 333, 452

Kühr, H., Witzel, A., Pauliny-Toth, I. I. K., \& Nauber, U. 1981, A\&AS, 45, 367

Lee, C.-U., Park, S. S., Kim, C. H., \& Byun, Y. I. 2003, JASS, 20, 143

Lloyd, C. 1984, MNRAS, 209, 697

Maesano, M., Massaro, E., \& Nesci, R. 1997, IAU Circ., 6700

Malkan, M., \& Moore, R. 1986, ApJ, 300, 216

Massaro, E., Nesci, R., Perola, G. C., Lorenzetti, D., \& Spinoglio, L. 1995, A\&A, 299, 339

Massaro, E., Nesci, R., Maesano, M., Montagni, F., \& D’Alessio, F. 1998, MNRAS, 299, 47

Mastichiadis, A., \& Kirk, J. G. 2002, PASA, 19, 138

Mattox, J., Wagner, S. J., Malkan, M., et al. 1997, ApJ, 476, 692

Mead, A. R. G., Ballard, K. R., Brand, P. W. J. L., et al. 1990, A\&AS, 83, 183

Mukherjee, R., Bertsch, D. L., Bloom, S. D., et al. 1997, ApJ, 490, 116

Nesci, R., Massaro, E., \& Montagni, F. 2002, PASA, 19, 143

Neshpor, Y. I., Stepanyan, A. A., Kalekin, O. P., et al. 1998, Astron. Lett., 24, 143

Penston, M. V., \& Cannon, R. O. 1970, R. Obs. Bull. 159, 85

Pian, E., Falomo, R., Hartman, R. C., et al. 2002, A\&A, 392, 407

Pian, E., Foschini, L., Beckmann, V., et al. 2005, A\&A, 429, 427

Pursimo, T., Takalo, L. O., Sillanpää, A., et al. 2000, A\&AS, 146, 141

Racine, R. 1970, ApJ, 159, L99

Raiteri, C. M., Villata, M., Aller, H. D., et al. 2001, A\&A, 377, 396

Raiteri, C. M., Villata, M., Tosti, G., et al. 2003, A\&A, 402, 151

Ramírez, A., de Diego, J. A., Dultzin-Hacyan, D., \& González-Pérez, J. N. 2004, A\&A, 421, 83

Romero, G. E., Cellone, S. A., \& Combi, J. A. 1999, A\&AS, 135, 477

Sagar, R., Gopal-Krishna, Mohan, V., et al. 1999, A\&AS, 134, 453

Sambruna, R. M., Aharonian, F. A., Krawczynski, H., et al. 2000, ApJ, 538,127

Schramm, K. J., Borgeest, U., Camenzind, M., et al. 1993, A\&A, 278, 391

Shen, B. S. P., \& Usher, P. D. 1970, Nature, 228, 1070

Sillanpää, A., Haarala, S., Valtonen, M. J., Sundelius, B., \& Byrd, G. G. 1988a, ApJ, 325, 628 
Sillanpää, A., Haarala, S., \& Korhonen, T. 1988b, A\&AS, 72, 347 Sillanpää, A., Takalo, L. O., Pursimo, T., et al. 1996a, A\&A, 305, L17 Sillanpää, A., Takalo, L. O., Pursimo, T., et al. 1996b, A\&A, 315, L13 Smith, A. G., Leacock, R. J., \& Webb, J. R. 1988, in Active Galactic Nuclei, ed. H. R. Miller, \& P. J. Wiita (Berlin: Springer), 307, 158 Smith, A. G., Nair, A. D., Leacock, R. J., \& Clements, S. D. 1993, AJ, 105,437

Smith, P. S., \& Balonek, T. J. 1998, PASP, 110, 1164

Smith, P. S., Balonek, T. J., Heckert, P. A., et al. 1985, AJ, 90, 1184

Tommasi, L., Palazzi, E., Pian, E., et al. 2001, A\&A, 376, 51

Ulrich, M. H., Maraschi, L., \& Urry, C. M. 1997, ARA\&A, 35, 445

Vagnetti, F., Trevese, D., \& Nesci, R. 2003, ApJ, 590, 123

Valtaoja, E., Teräsranta, H., Tornikoski, M., et al. 2000, ApJ, 531, 744

Villata, M., Raiteri, C. M., Ghisellini, G., et al. 1997, A\&AS, 121, 119

Villata, M., Raiteri, C. M., Lanteri, L., Sobrito, G., \& Cavallone, M. 1998a, A\&AS, 130, 305
Villata, M., Raiteri, C. M., Sillanpää, A., \& Takalo, L. O. 1998b, MNRAS, 293, L13

Villata, M., Mattox, J. R., Massaro, E., et al. 2000, A\&A, 363, 108

Villata, M., Raiteri, C. M., Sobrito, G., et al. 2001, ApL\&C, 40, 123

Villata, M., Raiteri, C. M., Kurtanidze, O. M., et al. 2002, A\&A, 390, 407

Villata, M., Raiteri, C. M., Kurtanidze, O. M., et al. 2004, A\&A, 421, 103

Wagner, S. J., Camenzind, M., Dreissigacker, O., et al. 1995, A\&A, 298, 688

Webb, J. R., Smith, A. G., Leacock, R. J., et al. 1988, AJ, 95, 374

Wehrle, A. E., Pian, E., Urry, C. M., et al. 1998, ApJ, 479, 178

Xie, G., Liang, E., Xie, Z., \& Dai, B. 2002, AJ, 123, 2352

Xie, G. Z., Zhou, S. B., Li, K. H., et al. 2004, MNRAS, 348, 831 